\title{
Structural Stability of Linear Dynamically Varying (LDV) Controllers
}

\author{
Stephan Bohacek and Edmond Jonckheere \\ Dept. of Electrical engineering - Systems \\ University of Sourthern California \\ Los Angeles, CA 90089-2563 \\ (213)-740-4457 \\ jonckhee@eudoxus.usc.edu
}

\begin{abstract}
Linear dynamically varying (LDV) controllers have been shown to be useful in controlling nonlinear dynamical systems on compact sets, especially chaotic systems. In this paper it is shown that the ability to stabilize a dynamical system with an LDV controller is structurally stable in the $C^{1}$ topology, the Lipschitz topology and, in a restricted sense, the $C^{0}$ topology provided that these systems are near enough to an LDV stabilizable $C^{1}$ dynamical system. Furthermore, the optimal LDV controller is shown to depend continuously on the dynamical system.
\end{abstract}

\section{Introduction}

Recently linear dynamically varying (LDV) systems were introduce as good linearized models of nonlinear dynamical systems on compact sets. Both $H_{\infty}[1]$ and optimal linear quadratic [2] controllers have been developed for such linear systems. In this paper the stabilizability of dynamical systems will be investigated. Specifically, suppose that the dynamical system $f$ is stabilizable in the sense of these LDV methods; what can we say about the stabilizability of dynamical systems $\hat{f}$ that are close to $f$ in different topologies? Furthermore, how does the optimal controller vary as the map $f$ varies?

An LDV system is defined as

$$
\begin{aligned}
x(k+1) & =A_{\theta(k)} x(k)+B_{\theta(k)} u(k) \\
z(k) & =\left[\begin{array}{c}
C_{\theta(k)} x(k) \\
D_{\theta(k)} u(k)
\end{array}\right] \\
\theta(k+1) & =f(\theta(k)) \quad \text { with } x(0)=x_{o}, \theta(0)=\theta_{o}
\end{aligned}
$$

where $f: S \rightarrow S$ is a continuous map; $A: S \rightarrow \mathbb{R}^{n \times n}$, $B: S \rightarrow \mathbb{R}^{n \times m}, C: S \rightarrow \mathbb{R}^{p \times n}, D: S \rightarrow \mathbb{R}^{p \times m} ;$ $S \subset \mathbb{R}^{n}$ is a compact set; and $S$ has the metric structure induced by the Euclidean metric. A continuous LDV is an LDV where the maps $A, B, C$ and $D$ are continuous. $0-7803-4394-8 / 98 \$ 10.00$ @ 1998 IEEE
Continuous LDV systems naturally arise when controlling nonlinear dynamical systems. Let

- $f: \mathbb{R}^{n} \times \mathbb{R}^{m} \rightarrow \mathbb{R}^{n}$

- $f \in C^{1}$,

- $f(S, 0)=S$,

- $S$ be compact.

Consider the nonlinear tracking problem:

$$
\begin{array}{rlc}
\varphi(k+1) & =f(\varphi(k), u(k)) & \varphi(0)=\varphi_{\circ} \\
\theta(k+1) & =f(\theta(k), 0) & \theta(0)=\theta_{\circ} \in S .
\end{array}
$$

The objective is to find a control $u$ such that $\|\varphi(k)-\theta(k)\| \rightarrow 0$. In this context $\{\theta(k): k \geq 0\}$ is the desired trajectory and $\varphi(k)$ is the state of the system under control. This tracking problem is common to many chaos control problems, see [2] and [1] for more details. Furthermore, if $f$ is invertible, then the adjoint of this tracking problem is an observer problem. The observer, or observer/controller problem is closely related to chaos synchronization [6]. This paper focuses on tracking; the dualization to the observer is left to the reader. The tracking error is defined as $x(k)=\varphi(k)-\theta(k)$ with error dynamics:

$$
\begin{aligned}
x(k+1)= & f(\varphi(k), u(k))-f(\theta(k), 0)) \\
= & A_{\theta(k)} x(k)+B_{\theta(k)} u(k) \\
& +\eta(x(k), u(k), \theta(k))
\end{aligned}
$$

where $\left(A_{\theta}\right)_{i, j}=\frac{\partial f_{i}}{\partial \theta_{j}}(\theta, 0),\left(B_{\theta}\right)_{i, j}=\frac{\partial f_{i}}{\partial u_{j}}(\theta, 0)$ and $\eta(x, u, \theta)=\eta_{x}(x, u, \theta) x+\eta_{u}(x, u, \theta) u$ with

$\eta_{x}(x, u, \theta)_{i, j}=\int_{0}^{1} \frac{\partial f_{i}}{\partial x_{j}}(t x+\theta, t u) d t-\frac{\partial f_{i}}{\partial x_{j}}(\theta, 0)$

$\eta_{u}(x, u, \theta)_{i, j}=\int_{0}^{1} \frac{\partial f_{i}}{\partial u_{j}}(t x+\theta, t u) d t-\frac{\partial f_{i}}{\partial u_{j}}(\theta, 0)$.

Since $f \in C^{1}$, if $x$ and $u$ are small, then $\eta_{x}(x, u, \theta)$ and $\eta_{u}(x, u, \theta)$ are small. Thus, when $x$ and $u$ are small, 
system 3 is well approximated by system 1 with $f(\theta):=$ $f(\theta, 0)$. In this case the LDV system 1 is said to be induced by $f$.

Robust stability is a important concept in control. A system is robust if it remains stable under small variations of the system. More generally, structural stability means that certain properties do not change under small variations of the system. Hence, a controller is robust if the stability of closed loop system is structurally stable. Similarly, the optimal linear quadratic or $H_{\infty}$ controller would be structurally stable if small variations in the system only lead to small variations in the controller. Thus structural stability is the absence of bifurcations. The structural stability of LDV systems is complicated by the fact that as $f$ varies not only do the system parameters $\left(A_{\theta}, B_{\theta}, C_{\theta}, D_{\theta}\right)$ vary for each $\theta \in S$ but as $k$ grows $\left(A_{f^{k}(\theta)}, B_{f^{k}(\theta)}, C_{f^{k}(\theta)}, D_{f^{k}(\theta)}\right)$ may greatly vary, for example $\left\|A_{f^{k}(\theta)}-A_{f^{k}(\theta)}\right\|$ may be large.

Incomplete knowledge of the map $f$ can arise in different ways. The map defining the desired trajectory may be known, while the map defining the system under control is only approximated known, i.e.

$$
\begin{aligned}
\varphi(k+1) & =\hat{f}(\varphi(k), u(k)) \\
\theta(k+1) & =f(\theta(k), 0)
\end{aligned}
$$

where $f$ is known and $\hat{f}$ is only known to be approximated by $f$. This system can be modeled as the following LDV system with noise:

$$
\begin{aligned}
x(k+1)= & \frac{\partial f}{\partial \theta}(\theta(k), 0) x(k) \\
& +\frac{\partial f}{\partial \theta}(\theta(k), 0) u(k)+w(k) \\
\theta(k+1)= & f(\theta(k), 0)
\end{aligned}
$$

where $w(k)=\hat{f}(\varphi(k), u(k))-f(\varphi(k), u(k))$ as is known to be small. Standard robustness of timevarying systems show that $x$ remains bounded if $w$ is small enough. A more complex situation occurs when the map defining the desired trajectory and the map defining the system under control are the same and only approximately known. In this case the tracking dynamics are

$$
\begin{aligned}
& x(k+1)=\frac{\partial \hat{f}}{\partial \theta}(\theta, 0) x(k)+\frac{\partial \hat{f}}{\partial u}(\theta, 0) u(k) \\
& \hat{\theta}(k+1)=\hat{f}(\hat{\theta}(k), 0)
\end{aligned}
$$

where $\hat{f}$ is known to be approximated by $f$. This is the scenario that will be investigated here. Other situations can be framed as combinations of 4 and 5 . That is, system 5 with some noise added.

This paper proceeds as follows: The next section provides background regarding LDV systems and different topologies on the space of dynamical systems, that is, what is meant by $f$ being approximated by $f$. In section 3 the stabilizability of conjugate dynamical systems is investigated. Section 4 presents the main results that stabilizability and optimal LDV controllers are structurally stable. And finally, in section 5, an example illustrates how the optimal LDV controller varies with the parameters of the Henon map.

\section{Background}

We say that the pair $(A, f)$ is exponentially stable if system 1 is exponentially stable. That is, for $u=0$ and $\theta_{o} \in S$ there exists an $\alpha\left(\theta_{o}\right)<1$ and a $\beta\left(\theta_{o}\right)<\infty$ such that if $\theta(0)=\theta_{o}$, then $\|x(k)\|<\beta\left(\theta_{o}\right) \alpha\left(\theta_{o}\right)^{k}\|x(0)\|$. Similarly, the pair $(A, f)$ is uniformly exponentially stable if the pair $(A, f)$ is exponentially stable and $\alpha$ and $\beta$ can be chosen independent of $\theta(0)$. The triple $(A, B, f)$ is stabilizable if there exists a bounded feedback $F: S \rightarrow \mathbb{R}^{m \times n}$ such that $(A+B F, f)$ is exponentially stable. Note that uniform exponential stability is not required for a system to be stabilizable. The triple $(A, C, f)$ is uniformly detectable if there is a uniformly bounded map $H: S \rightarrow R^{n \times p}$ such that $(A+H C, f)$ is uniformly exponentially stable. That is, there exists $\alpha_{d}<1$ and $\beta_{d}<\infty$ such that for all $\theta(0) \in S,\|x(k)\|<\beta_{d} \alpha_{d}^{k}\|x(0)\|$ where $x(k+1)=\left(A_{f^{k}\left(\theta_{0}\right)}+H_{f^{k}\left(\theta_{0}\right)} C_{f^{k}\left(\theta_{o}\right)}\right) x(k)$.

It was shown in [1] and [2] that if the LDV system 1 induced by $f$ is uniformly exponentially stabilized by the control $u(k)=F_{\theta(k)} x(k)$, then the nonlinear system 2 , with control $u(k)=F_{\theta(k)} x(k)$, is locally uniformly exponentially stable. By definition locally uniformly exponentially stable means that there exist $\alpha<1, \beta<\infty$ and $\gamma>0$ such that if $\|x(0)\|=\|\varphi(0)-\theta(0)\|<\gamma$ then $\|x(k)\|<\beta \alpha^{k}\|x(0)\|$ where $\alpha, \beta$ and $\gamma$ can be taken independent of the initial condition $\theta_{o}$, i.e. uniformly in $\theta_{o}$ and locally in $x$. Therefore, we say that the dynamical system $f$ is LDV stabilizable if the LDV system induced by $f$ is stabilizable. In section 3 an example shows that a dynamical system may be stabilizable but not LDV stabilizable.

The following theorem is needed in the sequel:

Theorem 1 Suppose 1 is a continuous, uniformly detectable $L D V$ system with $D_{\theta}^{\prime} D_{\theta}>0$ for $\theta \in S$. Then system 1 is stabilizable if and only if there exists a bounded function $X: S \rightarrow \mathbb{R}^{n \times n}$ with $X_{\theta}^{\prime}=X_{\theta} \geq 0$ that satisfies the functional discrete time algebraic Riccati equation

$$
\begin{aligned}
& X_{\theta}=A_{\theta}^{\prime} X_{f(\theta)} A_{\theta}+C_{\theta}^{\prime} C_{\theta} \\
& -A_{\theta}^{\prime} X_{f(\theta)} B_{\theta}\left(D_{\theta}^{\prime} D_{\theta}+B_{\theta}^{\prime} X_{f(\theta)} B_{\theta}\right)^{-1} B_{\theta}^{\prime} X_{f(\theta)} A_{\theta}
\end{aligned}
$$

In this case the control

$u_{L Q}(k)=F_{\theta(k)} x(k)$ 


$$
\begin{aligned}
= & -\left(D_{\theta(k)}^{\prime} D_{\theta(k)}+B_{\theta(k)}^{\prime} X_{f(\theta(k))} B_{\theta(k)}\right)^{-1} \\
& \times B_{\theta(k)}^{\prime} X_{f(\theta(k))} A_{\theta(k)}
\end{aligned}
$$

is optimal in the sense that it minimizes the quadratic cost

$$
\begin{aligned}
V\left(\theta_{o}, u, x_{o}\right)= & \sum_{k=0}^{\infty} x(k)^{\prime} C_{f^{k}\left(\theta_{o}\right)}^{\prime} C_{f^{k}\left(\theta_{o}\right)} x(k) \\
& --u(k)^{\prime} D_{f^{k}\left(\theta_{o}\right)}^{\prime} D_{f^{k}\left(\theta_{o}\right)} u(k) .
\end{aligned}
$$

Furthermore, this control uniformly exponentially stabilizes the system and $x_{o}^{\prime} X_{\theta_{o}} x_{o}=\min _{u} V\left(\theta_{o}, u, x_{o}\right)$ and $X$ is a continuous function.

Proof: See [2].

Note that this theorem implies that there exists an exponentially stabilizing feedback if and only if there exists a uniformly exponentially stabilizing feedback. Thus, when considering continuous LDV systems, stabilizability is equivalent to the existence of a uniformly exponentially stabilizing feedback.

Given a stabilizable map $f$, we will study maps close to $f$ in the following topologies:

Definition 1 Let $f, \hat{f}: \mathbb{R}^{n} \times \mathbb{R}^{m} \rightarrow \mathbb{R}^{n}$ with $f, \hat{f} \in C^{1}$. The $C^{\mathbf{1}}$ topology is generated by the metric

$$
\begin{aligned}
d_{C^{1}}(f, \hat{f})= & \sup _{\theta \in \mathbb{R}^{n}, u \in \mathbb{R}^{m}}\|f(\theta, u)-\hat{f}(\theta, u)\| \\
& +\sup _{\theta \in \mathbb{R}^{n}, u \in \mathbb{R}^{m}}\left\|\frac{\partial f}{\partial \theta}(\theta, u)-\frac{\partial \hat{f}}{\partial \theta}(\theta, u)\right\| \\
& +\sup _{\theta \in \mathbb{R}^{n}, u \in \mathbb{R}^{m}}\left\|\frac{\partial f}{\partial u}(\theta, u)-\frac{\partial \hat{f}}{\partial u}(\theta, u)\right\|
\end{aligned}
$$

where $\frac{\partial f}{\partial \theta}(\theta, u)$ is the Jacobian matrix of $f$ with respect to $\theta$ and $\|\cdot\|$ is the $l_{2}$ induced matrix norm.

Definition 2 Let $f, \hat{f}: \mathbb{R}^{n} \times \mathbb{R}^{m} \rightarrow \mathbb{R}^{n}$, with $f, \hat{f} \in$ $L C$, where $L C$ denotes the set of Lipschitz continuous functions. The LC topology is generated by the metric

$$
\begin{gathered}
d_{L C}(f, \hat{f})=\sup _{\theta \in \mathbb{R}^{n}, u \in \mathbb{R}^{m}}\|f(\theta, u)-\hat{f}(\theta, u)\| \\
\sup \left\{\frac{\left|f_{i}(\theta, u)-f_{i}(\varphi, v)-\left(\hat{f}_{i}(\theta, u)-\hat{f}_{i}(\varphi, v)\right)\right|}{\sqrt{\|\theta-\varphi\|^{2}+\|u-v\|^{2}}}\right. \\
\left.: \theta, \varphi \in \mathbb{R}^{n}, u, v \in \mathbb{R}^{m}, i \in[1, n]\right\} .
\end{gathered}
$$

Definition 3 Let $f, \hat{f}: \mathbb{R}^{n} \times \mathbb{R}^{m} \rightarrow \mathbb{R}^{n}$, with $f, \hat{f} \in$ $C^{0}$. The $C^{0}$ topology is generated by the metric,

$$
d_{C^{\circ}}(f, \hat{f})=\sup _{\theta \in \mathbb{R}^{n}, u \in \mathbb{R}^{m}}\|f(\theta, u)-\hat{f}(\theta, u)\| .
$$

Remark 1 The supremums in the last three definitions are over $\theta \in \mathbb{R}^{n}$ and $u \in \mathbb{R}^{m}$. This can be eased to the supremum over $\mathcal{N}$ where $\mathcal{N} \subset \mathbb{R}^{n} \times \mathbb{R}^{m}$ is a tubular neighborhood of $S \times\{0\}$. This modification has no effect on the development that follows if $\mathcal{N}$ is large enough, i.e. for all $\theta \in S, \sup _{\varphi \in E_{\theta}}\|\varphi-\theta\|$ is large enough, where $E_{\theta}:=\left\{\varphi \in \mathcal{N}: \theta:=\arg \min _{v \in S}\|\varphi-v\|\right\}$. If $\mathcal{N}$ is not large enough, then minor changes are necessary. In particular, the initial error $x(0)$ must be smaller.

In the definition of system 1 , the set $S$ is invariant, i.e. $f(S)=S$. As the map $f$ varies it is likely that $S$ is no longer invariant. Indeed, it is possible that arbitrarily small variations in the map $f$ lead to drastic changes in invariant sets. This is problematic since $X$, the solution to the Riccati equation 6 , is only defined on $S$ where $S$ is invariant. It is difficult to discuss the dependence of $X$ on $f$ if as $f$ varies the domain of $X$ greatly varies. Thus we will restrict our attention to variations in $f$ such that $S$ only varies slightly. That is, we will require

$$
\begin{aligned}
& d .(f, \hat{f})+H(S, \hat{S})<\varepsilon \\
& f(S)=S, \hat{f}(\hat{S})=\hat{S}
\end{aligned}
$$

where $H(\cdot, \cdot)$ is the Hausdorff metric, i.e.

$$
\begin{aligned}
H(S, \hat{S}):= & \max \left(\sup _{\theta \in \hat{S}} \inf \{\|\theta-\hat{\theta}\|: \hat{\theta} \in \hat{S}\}\right. \\
& \left., \sup _{\hat{\theta} \in \hat{S}} \inf \{\|\theta-\hat{\theta}\|: \theta \in S\}\right)
\end{aligned}
$$

In the sequel it will be understood that $S$ is an invariant set of $f$ and $\hat{S}$ is an invariant set of $\hat{f}$.

Next we extend the feedback $F: S \rightarrow \mathbb{R}^{n \times m}$ defined by equation 7 to all of $\mathbb{R}^{n}$ by

$$
\tilde{F}_{\varphi}:=F_{\theta(\varphi)}
$$

where

$$
\theta(\varphi)=\arg \min \{\|\theta-\varphi\|: \theta \in S\}
$$

The cost quadratic $X$ can be extended to $\tilde{X}$ in the same fashion. Note that $\theta(\varphi)$ is not necessarily well defined and $\tilde{X}$ may not be continuous. However, by perhaps invoking the axiom of choice one can properly define $\theta(\varphi)$. Furthermore, $\tilde{X}$ is continuous on $S$ and if $\|\hat{\theta}-\hat{\varphi}\|$ is small and $\hat{\theta}, \hat{\varphi} \in \hat{S} \cup S$ with $H(S, \hat{S})$ small, then $\left\|\tilde{X}_{\hat{\theta}}-\tilde{X}_{\hat{\varphi}}\right\|$ is small. Finally, let $X: S \rightarrow \mathbb{R}^{n}$ and $\hat{X}: \hat{S} \rightarrow \mathbb{R}^{n} ;$ define

$$
\begin{aligned}
& d_{S, \hat{S}}(X, \hat{X}):= \\
& \max \left(\sup _{\theta \in S}\left\|X_{\theta}-\hat{X}_{\hat{\theta}(\theta)}\right\|, \sup _{\hat{\theta} \in \hat{S}}\left\|X_{\theta(\hat{\theta})}-\hat{X}_{\hat{\theta}}\right\|\right)
\end{aligned}
$$


Now if $f$ is a hyperbolic on $S$ (see section 3), then $H(S, \hat{S})$ is small when $d_{C^{1}}(f, \hat{f})$ is small [5]. Furthermore, if $f: S \rightarrow S$ is hyperbolic on $S$ and $S$ is a manifold (i.e. $f$ is an Anosov diffeomorphism) and $\hat{f}: S \rightarrow S$, then $\hat{f}$ is hyperbolic on $\hat{S}$ and $\hat{S}$ is invariant when $d_{C^{1}}(f, \hat{f})$ is small enough. Therefore, if $f$ is an Anosov diffeomorphism, $H(S, \hat{S})=0$ and $d_{S, \hat{s}}(X, \hat{X})=d_{C^{0}}(X, \hat{X})$. On the other hand, suppose that $S$ is an attractor for $f$, that is for all $\varphi \in \mathcal{N}(S)$, we have $\lim f^{k}(\varphi) \subset S$ where $\mathcal{N}(S)$ is any small enough neighborhood of $S$. Then for a generic $f$, $H(S, \hat{S})$ is small when $d_{C^{\circ}}(f, \hat{f})$ is small [7], where $\hat{S}$ is an attractor for $\hat{f}$. Hence, in these three cases $d_{C^{1}}(f, \hat{f})$ small implies $H(S, \hat{S})$ is small, and therefore condition 8 is repetitious. Nonetheless, to maintain generality, condition 8 will be assumed.

\section{Conjugacy}

Let $f$ be LDV stabilizable and let $f$ and $\hat{f}$ be $C^{1}$ conjugate. That is, there exists diffeomorphisms

$$
g: \mathbb{R}^{n} \rightarrow \mathbb{R}^{n}, g(S)=\hat{S}
$$

and

$$
h: \mathbb{R}^{m} \rightarrow \mathbb{R}^{m}
$$

such that $f(\theta, u)=g^{-1}(\hat{f}(g(\theta), h(u)))$. Define $G(\theta)=\frac{\partial g}{\partial \theta}(\theta), H=\frac{\partial h}{\partial u}(0)$. Since $g$ and $h$ are diffeomorphisms, $G(\theta)$ and $H$ are invertible matrices. Therefore the following diagram commutes:

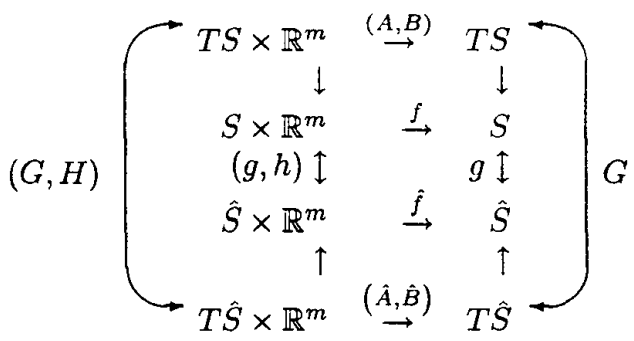

Thus, if $(A, B, f)$ is the LDV system induced by $f$ and $(\hat{A}, \hat{B}, \hat{f})$ is the LDV system induced by $\hat{f}$, then $\hat{A}_{g(\theta)}=G_{f(\theta)} A_{\theta} G_{g(\theta)}^{-1}, \hat{B}_{g(\theta)}=G_{f(\theta)} B_{\theta} H_{g(\theta)}^{-1}$. That is, conjugacy lead to an invertible change of coordinates. Therefore, if $F: S \rightarrow \mathbb{R}^{m \times n}$ uniformly exponentially stabilizes $(A, B, f)$, then $H_{\theta} F_{\theta} G_{g(\theta)}^{-1}=\hat{F}_{g(\theta)}$ uniformly exponentially stabilizes $(\hat{A}, \hat{B}, \hat{f})$. Thus, LDV stabilizability is preserved under $C^{1}$ conjugacy. Similarly, LDV uniformly detectability is preserved under $C^{1}$ conjugacy.

Suppose $f$ and $\hat{f}$ are $C^{1}$ conjugate, $(A, B, f)$ is stabilizable, $(A, C, f)$ is uniformly detectable, $D_{\theta}^{\prime} D_{\theta}>0$ for $\theta \in S, \hat{C}_{g(\theta)}=C_{\theta} G_{g(\theta)}^{-1}$ and $\hat{D}_{g(\theta)}=D_{\theta} H_{\theta}^{-1}$. Since $f \in C^{1}$, the LDV system induced by $f$ is continuous and theorem 1 implies that there exists a continuous function $X: S \rightarrow \mathbb{R}^{n \times n}$ that solves equation 6 . It is easy to show that $\hat{X}_{g(\theta)}:=\left(G_{g(\theta)}^{-1}\right)^{\prime} X_{\theta} G_{g(\theta)}$ solves the Riccati equation associated with the LDV system induced by $\hat{f}$. Therefore, LDV systems and optimal controllers are well defined in a coordinate free approach. The topological and geometric issues associated with LDV systems on a non-orientable or non-parallelizable manifolds $S$ are addressed in [4].

Now, suppose that $f$ is hyperbolic on $S$. That is, there exists an $\varepsilon>0$ such that for all $\theta \in S$, there are $\mathbb{E}_{\theta}^{ \pm}$with $\mathbb{R}^{n}=\mathbb{E}_{\theta}^{+} \oplus \mathbb{E}_{\theta}^{-}, \frac{d f}{d \theta}(\theta, 0): \mathbb{E}_{\theta}^{ \pm} \rightarrow \mathbb{E}_{\theta}^{ \pm}$, $\operatorname{Re} \lambda\left(\left.\frac{d f}{d \theta}(\theta, 0)\right|_{\mathbb{E}_{\theta}^{+}}\right)>\varepsilon$ and $\operatorname{Re} \lambda\left(\left.\frac{d f}{d \theta}(\theta, 0)\right|_{\mathbb{E}_{\theta}^{-}}\right)<$ $-\varepsilon$, where $\operatorname{Re} \lambda\left(\left.A\right|_{\mathbb{E}}\right)>\varepsilon$ means that all of the eigenvalues of $A$ with eigenvectors in $\mathbb{E}$ have a real part that is greater than $\varepsilon$. If $f$ is hyperbolic and $\hat{f}$ is $C^{1}$ close to $f$, then $\hat{f}$ is hyperbolic and $\hat{f}$ is topologically conjugate to $f$ (for exact result see [5]). However, $f$ and $\hat{f}$ are not necessarily $C^{1}$ conjugate. If hyperbolicity implied $\hat{f}$ and $f$ are $C^{1}$ conjugate, then LDV stabilizability would clearly be structurally stable in the hyperbolic case. Note that in the case of topological conjugacy the nonlinear control $\hat{u}(k)=$ $h^{-1}\left(F_{\theta(k)}\left(g^{-1}(\varphi(k))-g^{-1}(\theta(k))\right)\right)$ might not exponentially stabilize $\hat{f}$. Consider, for example, a system with no input:

$$
f(\varphi)=\frac{1}{2} \varphi, \quad S=\left[0, \frac{1}{2}\right]
$$

and the induced LDV system

$$
x(k+1)=\frac{1}{2} x(k)
$$

Under homemorphisms

$$
g(\varphi)=\frac{-1}{\log (\varphi)} \text { and } g^{-1}(w)=e^{-\frac{1}{w}},
$$

the conjugate system is

$$
f(w)=\frac{1}{1-w \log \left(\frac{1}{2}\right)} w, \quad S=\left[0, \frac{-1}{\log \left(\frac{1}{2}\right)}\right]
$$

and the induced LDV system is

$$
\begin{aligned}
& \hat{x}(k+1)=\left(\frac{1}{(1+w(k) \ln 2)^{2}}\right) \hat{x}(k) \\
& w(k+1)=\hat{f}(w(k))
\end{aligned}
$$

The above system is not uniformly exponentially stable because for every $\beta<\infty$ and $\alpha<1,\|\hat{x}(k)\|>$ $\beta \alpha^{k}\|\hat{x}(0)\|$ for some $k$. Of course $\hat{f}^{k}(w) \rightarrow 0$, just not 
exponentially fast. Thus $f$ and $\hat{f}$ are topologically conjugate, 12 is LDV stabilizable and 11 is not. Therefore, since hyperbolicity only leads to topological conjugacy, hyperbolicity will not help to prove the $C^{1}$ structural stability of LDV stabilizability. We must rely on the fact that $f$ and $\hat{f}$ are $C^{1}$ close to infer LDV stabilizability of $\hat{f}$.

Remark 2 The example above illustrates the weakness of the LDV approach over nonlinear methods. The $L D V$ approach implies that $\hat{f}$ is not stable, when, in fact, it is stable.

\section{Structural Stability}

In this section it will be shown that if $\hat{f}$ is near an LDV stabilizable $f$ in the $C^{1}$ topology and $H(S, \hat{S})$ is small, then $\hat{f}$ is LDV stabilizable (proposition 3 ). In fact, the LDV optimal quadratic cost varies continuously with $d_{C^{1}}(f, \hat{f})+H(S, \hat{S})$ - that is the map $(f, S) \mapsto X$ is continuous where $X$ is the positive semidefinite function which solves equation 6 (proposition 5). Furthermore, if $\hat{f}$ is near $f$ in the Lipschitz topology and $H(S, \hat{S})$ is small, then $\hat{f}$ is also LDV stabilizable (proposition 6). Finally, if $\hat{f}$ is near $f$ in the $C^{0}$ topology and $H(S, \hat{S})$ is small, then an LDV controller may only stabilize $\hat{f}$ in the sense that $\limsup _{k}\|x(k)\|<\varepsilon$, where the control objective is $x(k) \rightarrow 0$ (proposition $7)$.

Lemma 2 Let system 1 be a continuous LDV. If the $\operatorname{pair}(A, f)$ is uniformiy exponentially stable, then there exists a $\delta>0$ such that if $d_{C^{\circ}}(f, \hat{f})+d_{C^{\circ}}(A, \hat{A})+$ $H(S, \hat{S})<\delta$, then the pair $(\hat{A}, \hat{f})$ is uniformly exponentially stable. Furthermore, the $\alpha$ and $\beta$ in the definition of uniformly exponential stability can be taken to only depend on $A, f$ and $\delta$.

Note, this lemma is only examining LDV systems and therefore does not require that $A=\frac{\partial f}{\partial \theta}$.

Proposition 3 Assume $f \in C^{1}$ induces a stabilizable LDV system, that is, there exists a continuous map $F: S \rightarrow \mathbb{R}^{m \times n}$ such that $(A+B F, f)$ is uniformly exponentially stable. Then there exists a $\delta>0$ such that if $d_{C^{1}}(f, \hat{f})+H(S, \hat{S})<\delta$, then $\hat{f}$ is $L D V$ stabilizable and is stabilized by the feedback $\tilde{F}$ where $\tilde{F}$ is the extension of $F$ as defined by equation 9. Furthermore, with this feedback $\tilde{F}$, the $\alpha, \beta$ and $\gamma$ in the definition of locally uniformly exponentially stability can be chosen to depend only on $f, F$ and $\delta$. Thus LDV stabilizability is a structurally stable property.

Thus, if the feedback $F$ stabilizes $f$, then $\tilde{F}$ stabilizes any function $\hat{f}$ near $f$ in the $C^{1}$ topology. A natural question is, how good of a controller is $\tilde{F}$ ? For instance, if $F$ is the LDV quadratic controller for the LDV system induced by $f$, how far is $\tilde{F}$ from the LDV quadratic controller for $\hat{f}$. That is, are LDV quadratic controllers structurally stable? First, note that uniform detectability is a structurally stable property. That is:

Lemma 4 Assume that $A, C$ and $f$ are continuous and $(A, C, f)$ is uniformly detectable. In this case there exists a $\delta>0$, such that if $d_{C^{0}}(f, \hat{f})+d_{C^{0}}(A, \hat{A})+$ $d_{C^{\circ}}(C, \hat{C})+H(S, \hat{S})<\delta$, then $(\hat{A}, \hat{C}, \hat{f})$ is uniformly detectable. That is, there exists $a \hat{\alpha}_{d}<1$, $\hat{\beta}_{d}<\infty$ and a feedback $\hat{H}$ such that $\|\xi(k)\|<$ $\hat{\beta}_{d} \hat{\alpha}_{d}^{k}\|\xi(0)\|$ where $\xi(k+1)=(\hat{A}+\hat{H} \hat{C}) \xi(k)$. Furthermore, $\hat{\alpha}_{d}$ and $\hat{\beta}_{d}$ only depend on $f, \delta$ and $\alpha_{d}$ and $\beta_{d}$ that are implied by the uniform detectability of $(A, C, f)$.

Proposition 5 Let $f \in C^{1}$. Assume that the $L D V$ system induced by $f$ is stabilizable, $(A, C, f)$ is uniformly detectable and for all $\theta \in S, D_{\theta}^{\prime} D_{\theta}>0$. Then for all $\varepsilon>0$, there exists $a \delta>0$ such that if $d_{C^{1}}(f, \hat{f})+d_{C^{\circ}}(C, \hat{C})+d_{C^{\circ}}(D, \hat{D})+H(S, \hat{S})<\delta$, then $d_{S, \hat{s}}(X, \hat{X})<\varepsilon$, where $X$ is the positive semidefinite solution to the Riccati equation 6 induced by $(C, D, f)$ and $\hat{X}$ is the solution induced by $(\hat{C}, \hat{D}, \hat{f})$.

Next we weaken the assumptions in proposition 3 . We examine the case where $\hat{f} \in L C$ with $d_{L C}(f, \hat{f})<\delta$, and find that proposition 3 still hold.

Proposition 6 Let $f \in C^{1}$ induce a stabilizable $L D V$. Then there exists an $\delta>0$ such that if $\hat{f} \in L C$ and $d_{L C}(f, \hat{f})+H(S, \hat{S})<\delta$, then the LDV controller induced by $f$ locally uniformly exponentially stabilizes $\hat{f}$.

If we only restrict $d_{C^{0}}(f, \hat{f})<\delta$, then we cannot guarantee asymptotic stability. For example, consider the dynamical system $f(\varphi, u)=\frac{1}{2} \varphi$. Then $f$ is globally uniformly exponentially stable with $|\varphi(k)-\theta(k)| \leq$ $\frac{1}{2}^{k}|\varphi(0)-\theta(0)|$. Define $\hat{f}(\varphi, u)=\frac{1}{2} \varphi+\delta \sin \left(\frac{\varphi}{\delta} \frac{\pi}{6}\right)$. Then $d_{C^{\circ}}(f, \hat{f}) \leq \delta$ and $\hat{f}$ has three fixed points corresponding to the solutions to $\sin \left(\frac{\varphi}{\delta} \frac{\pi}{6}\right)=\frac{\varphi}{2 \delta}$. Hence, 
$\pm \delta$ are stable fixed points and zero is an unstable fixed point. Thus if $\varphi>0$ and $\theta<0$, then $|\varphi(k)-\theta(k)| \nrightarrow$ 0 . However, $\lim \sup |\varphi(k)-\theta(k)|<2 \delta$. Thus $\hat{f}$ is stable; not asymptotically stable, but asymptotically $\varphi(k)-\theta(k)$ approaches a small set around zero. This form of stability is often referred to as asymptotically bounded and the attractive set that $\varphi(k)-\theta(k)$ enters is called a residual set.

Proposition 7 Let $f \in C^{1}$ be LDV stabilizable via the feedback $F$. Then there exists an $\delta>0$ such that if $d_{C^{\circ}}(f, \hat{f})+H(S, \hat{S})<\delta$, then the feedback $F$ makes $\hat{f}$ asymptotically bounded, with the diameter of the resid$u a l$ set continuous in $\delta$.

Remark 3 Typically one assumes that control methods of nonlinear maps based on linear approximation are only applicable to differentiable maps. However, the last two propositions show that this assumption is not necessary.

\section{Structural Stability of the Optimal LDV Controller of the Hénon Map}

The Hénon map is defined as

$$
\begin{aligned}
\varphi(k+1) & =f(\varphi(k), u(k)) \\
& =\left[\begin{array}{l}
1-(a+u(k))\left(\varphi_{1}(k)\right)^{2}+\varphi_{2}(k) \\
b \varphi_{1}(k)
\end{array}\right] .
\end{aligned}
$$

The LDV approximation of this system is:

$$
\begin{aligned}
x(k+1)= & {\left[\begin{array}{ll}
-2 a \theta_{1}(k) & 1 \\
b & 0
\end{array}\right] x(k) } \\
& +\left[\begin{array}{l}
\left(\varphi_{1}(k)\right)^{2} \\
0
\end{array}\right] u(k) \\
\theta(k+1)= & f(\theta(k), 0) .
\end{aligned}
$$

The Hénon map has been studied for a wide variety of parameters. It was first introduced with $a=1.4$ and $b=0.3[3]$. With these parameters it is not yet known if this system is chaotic. However, computer simulations show that the system has an attractor. An LDV controller for the Henon map was found in [2]. According to proposition 5 the optimal controller should not change too drastically for small changes in the parameters if the attractor does not change to much. However, the Hénon map is a very difficult since it is not structurally stable. For example, if $a=1.392$ and $b=0.3$, then the attractor $S$ is non-trivial with aperiodic orbits and the map appears to be chaotic. However, there are parameters $\hat{a}$ arbitrarily close to 1.392 such that the attractor is simply an infinite set of periodic orbits [8]. Figure 1 shows $\log \left(d_{C^{1}}(f, \hat{f})+H(S, \hat{S})\right)$ versus

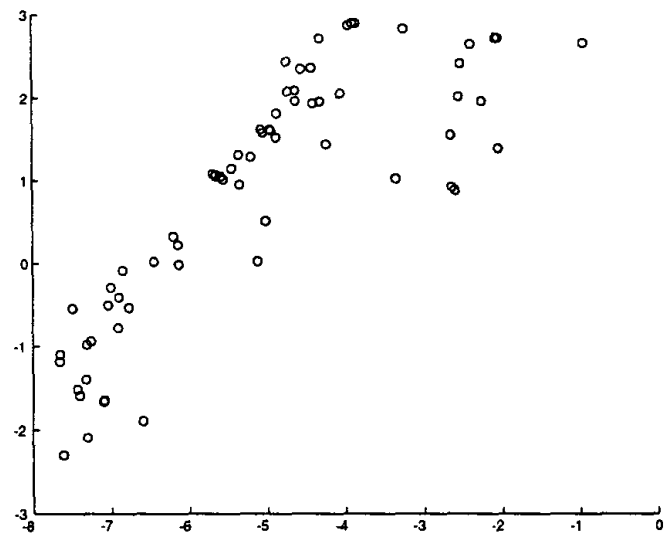

Figure 1: See text for discussion.

$\log \left(d_{S, \dot{S}}(X, \hat{X})\right)$ where $X$ is the solution to the functional algebraic Riccati equation 6 with $a=1.4$ and $b=0.3$ and $\hat{X}$ is the solution for other values of $a$ and $b$. Note that as predicted by proposition $5, d_{S, \hat{S}}(X, \hat{X})$ is small when $d_{C^{1}}(f, \hat{f})+H(S, \tilde{S})$ small.

\section{References}

[1] S. Bohacek and E. Jonckheere. Linear dynamically varying h-infinity control of chaos. To be submitted.

[2] S. Bohacek and E. Jonckheere. Controlling chaos with chaotically varying linear quadratic control. Submitted for publication, submitted July 1997.

[3] M. Henon. A two-dimensional mapping with a strange attractor. Comm. Math. Phys., 50:69-77, 1976.

[4] E. Jonckheere and S. Bohacek. Global topological aspects of continuous-time linear dynamically varying (ldv) control. In 37th Conference on Decision and Control, 1998.

[5] A. Katok and B. Hasselblatt. Introduction to the Modern Theory of Dynamical Systems. Cambridge University Press, 1995.

[6] H. Nijmeijer and I. M. Y. Mareels. An observer looks at synchronization. IEEE Transactions on $C i$ cuits and Systems I, 44:882-890, 1997.

[7] S. Y. Pilyugin. The Space of Dynamical Systems with the $C^{0}$-Topology. Springer-Verlag, 1991.

[8] C. Robinson. Bifurcation to infinitely many sinks. Comm. Math. Phys., 90:433-459, 1983. 